\title{
US ED Opioid-Related Visits Increase, While Use of Medication for Opioid Use Disorder Undetectable, 2011-2016
}

\author{
Siri Shastry, $M D^{7} \mathbb{D}^{\mathrm{D}}$, Alex F. Manini, MD, MS², Lynne D. Richardson, $M D^{7}$, and \\ Michelle P. Lin, MD, MPH, $M S^{\top}$
}

'Department of Emergency Medicine, Icahn School of Medicine at Mount Sinai, Mount Sinai Hospital, New York, NY, USA; ${ }^{2}$ Division of Medical Toxicology, Department of Emergency Medicine, Icahn School of Medicine, Elmhurst Hospital Center, New York, NY, USA.

J Gen Intern Med 35(3):965-6

DOI: $10.1007 / \mathrm{s} 11606-019-05249-3$

(c) Society of General Internal Medicine 2019

\section{BACKGROUND}

Opioid use disorder (OUD) and the opioid epidemic have been declared a national emergency in the USA with nearly 400,000 deaths from 1999 to 2016. ${ }^{1}$ Emergency Departments (EDs) often serve as the first point of contact for patients with OUD with ED visits for overdose increasing by over $30 \%$ from 2016 to $2017 .^{2}$ ED initiation of medication for opioid use disorder (MOUD), specifically buprenorphine, with linkage to outpatient follow-up, is known to increase engagement with outpatient addiction treatment and reduce self-reported illicit opioid use. $^{2,3}$

However, trends in ED MOUD utilization since 2011 are unknown. ${ }^{4}$ Given the emergence of evidence supporting EDinitiated buprenorphine induction since $2015,2,3$ an updated examination is needed to better understand current practice patterns.

\section{METHODS}

We performed a retrospective analysis to describe trends in ED buprenorphine administration or prescription among opioidrelated ED visits using the 2011-2016 National Hospital Ambulatory Medical Care Survey (NHAMCS) and opioid-related outpatient visits from National Ambulatory Medical Care Survey (NAMCS) as a comparator. We also examined trends in other MOUD (methadone and naltrexone).

We used previously validated ICD-9 or ICD-10 codes corresponding to primary diagnosis related to $\mathrm{OUD}^{5}$ to calculate opioid-related visits. We included visits resulting in discharge only in order to focus on ED administration or prescription with planned outpatient follow-up, as recommended by recent

Previous Presentation An abstract of this study was presented at the Society of Academic Emergency Medicine Mid-Atlantic Regional Meeting: March 30, 2019; Washington, D.C. as well as the Society of Academic Emergency Medicine Annual Meeting: May 15, 2019; Las Vegas, NV.

Published online August 14, 2019 studies. Buprenorphine use was identified using the variable "medications administered or prescribed." All medication administrations were analyzed and medication administration frequencies were tabulated using the Multum nomenclature.

Unweighted and population-weighted frequencies for all opioid-related visits were calculated accounting for the survey-weighted design; visits by patients under age 18 and those resulting in hospital admission were excluded. US census data for each corresponding year was employed to compute visit rates per 100,000. Unweighted and weighted medication administration frequencies of MOUD were calculated. Descriptive statistics were generated and frequencies were tabulated using SAS University Edition [Version 9.4m5].

\section{RESULTS}

Opioid-related visit rates increased by $41.8 \%$ from 2011 to 2016, from 203.8 per 100,000 to 289 per 100,000 in 2016. Over the same time period, there were fewer than thirty recorded administrations of buprenorphine, methadone, or naltrexone across all years (Fig. 1a). Because population weights for events with unweighted frequencies below 30 are unreliable, ${ }^{6}$ we were unable to calculate weighted frequencies. In comparison, from NAMCS data, outpatient utilization of buprenorphine steadily increased from 2011 to 2016 in NAMCS, from 8,367,931 weighted administrations in 20112012 to $11,348,466$ weighted administrations in 2015-2016, representing a $35 \%$ relative increase (Fig. 1b).

\section{DISCUSSION}

In this nationally representative sample of ED visits, utilization of all types of MOUD, including buprenorphine, has remained undetectable, with no appreciable increase in administration and prescription from 2011 to 2016 despite opioid-related visits steadily increasing by nearly $42 \%$ over the same time period. In contrast, outpatient utilization of buprenorphine has substantially increased with a $35 \%$ increase from 2011 to 2016. In light of the nationwide burden of OUD, our findings suggest an important 


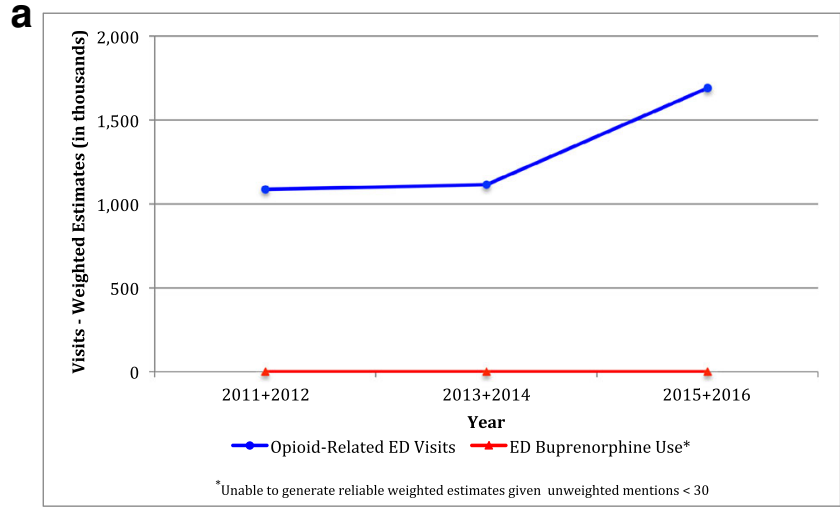

b

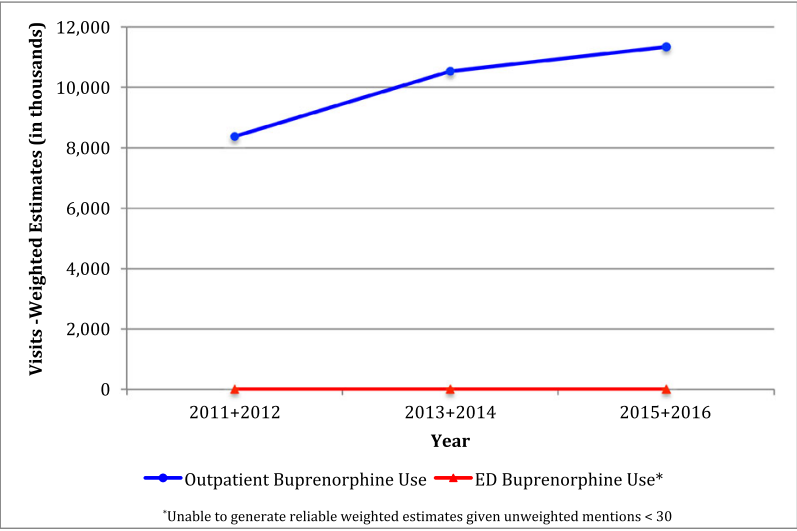

Figure 1 a US opioid-related ED visits and BUP use, 2011-2016. b ED and outpatient BUP use, 2011-2016.

public health gap. The fact that buprenorphine utilization has increased in outpatient clinic settings, while ED utilization remains undetectable, is of particular concern because the ED serves as a safety net for vulnerable populations who may particularly benefit from MOUD. Further research investigating barriers to ED administration and prescription of MOUD from the ED-including barriers to the expansion of buprenorphine prescription waivers among ED clinicians, prescription buprenorphine coverage, and access to outpatient follow-up - are urgently needed.

Our study is limited by the administrative nature of the data, including potential inconsistencies in ICD-9 or ICD10 diagnosis for opioid-related visits, and by changes in survey sampling approach between years, resulting in year-to-year variation in response rates and medication counts.

\section{Acknowledgments:}

The authors wish to thank Dr. Ethan A. Cowan, MD, MS (Department of Emergency Medicine, Icahn School of Medicine at Mount Sinai, New York, NY) for his involvement in the revision of this manuscript.

Funding/Support: Dr. Shastry is supported by an institutional training grant, 1 T32 HL129974-01 (PI: Richardson), from the National Heart, Lung \& Blood Institute of the National Institutes of Health.

Corresponding Author: Siri Shastry, MD; Department of Emergency Medicine, Icahn School of Medicine at Mount Sinai Mount Sinai Hospital, New York, NY, USA (e-mail: Siri.Shastry@mountsinai.org).

Author Contributions Dr. Shastry and Dr. Lin had full access to all of the study data, which are publically available from the National Center for Health Statistics. Study concept and design: Shastry and Lin. Acquisition of data: Shastry. Analysis and interpretation of data: Shastry and Lin. Drafting of the manuscript: Shastry and Lin. Critical revision of the manuscript: Manini and Richardson. Statistical analysis: Shastry and Lin. Obtained funding: Richardson. Study supervision: Lin, Manini, and Richardson.

\section{Compliance with Ethical Standards:}

Conflict of Interest: The authors declare that they do not have a conflict of interest.

Disclaimer: The content is solely the responsibility of the authors and does not necessarily represent the official views of the National Institutes of Health.

\section{REFERENCES}

1. Scholl L, Seth P, Kariisa M, Wilson N, Baldwin G. Drug and OpioidInvolved Overdose Deaths - United States, 2013-2017. WR Morb Mortal Wkly Rep. 2018. Accessed February 3, 2019.

2. D'Onofrio G, McCormack RP, Hawk K. Emergency departments - a 24/ 7/365 option for combating the opioid crisis. N Engl J Med. 2018;379(26):2487-2490.

3. D'Onofrio G, O'Connor PG, Pantalon MV, et al. Emergency departmentinitiated buprenorphine/naloxone treatment for opioid dependence: a randomized clinical trial. JAMA. 2015;313(16): 1636-44

4. Crane EH. Emergency Department Visits Involving Buprenorphine. The CBHSQ Report. Rockville: Substance Abuse and Mental Health Services Administration (US); 2013.

5. Moore BJ, Barrett ML. Case Study: Exploring How Opioid-Related Diagnosis Codes Translate From ICD-9-CM to ICD-10-CM. ONLINE. April 24, 2017. U.S. Agency for Healthcare Research and Quality. Available: https://www.hcupus.ahrq.gov/datainnovations/icd10_resources.jsp. Accessed 12 Nov 2018.

6. McCaig LF, Burt CW. Understanding and interpreting the National Hospital Ambulatory Medical Care Survey: key questions and answers. Ann Emerg Med. 2012;60(6)716-721.

Publisher's Note Springer Nature remains neutral with regard to jurisdictional claims in published maps and institutional affiliations. 